\title{
COMPUTING TRANSFER FUNCTIONS OF MULTIPOLE DEVICES DIRECTLY FROM MAGNETIC FIELD DATA, INCLUDING FRINGE FIELD EFFECTS AND HIGHER ORDER ABERRATIONS
}

\author{
R.M.G.M. Trines ${ }^{1,2}$, J.I.M. Botman ${ }^{2}$, S.J.L. van Eijndhoven ${ }^{1}$ \\ H.L. Hagedoorn ${ }^{2}$, T.J. Schep ${ }^{3}$
}

\section{Abstract}

The scalar and vector potentials of a magnetic multipole device are described in terms of magnetic field measurements gathered on either a cylindrical surface or a median plane [1]. Fringe field effects and multipole contributions of arbitrary order, as well as the discrete nature of the field measurements, are taken into account. This description has been used to calculate the transfer function of the device, directly in terms of the field measurements. The method as described in this paper can be applied to single beam guiding elements as well as to clusters of elements, and can be extended to calculate the transfer function for a complete beam line setup or a storage ring. The accuracy of the results is only limited by the accuracy of the field measurements.

\section{INTRODUCTION}

From Maxwell's equations for the static electromagnetic field, we find that, for a magnetic field $\vec{B}$ in a region without free charges or currents, there exists a scalar potential $u$, and a vector potential $\vec{A}$, satisfying:

$$
\begin{array}{r}
\operatorname{grad} u=\vec{B}=\operatorname{curl} \vec{A}, \\
\Delta u=0, \\
\text { curl curl } \vec{A}=\overrightarrow{0} .
\end{array}
$$

The vector potential $\vec{A}$ will be chosen such that $\operatorname{div} \vec{A}=0$. In this case, we have $\Delta \vec{A}=\overrightarrow{0}$.

We apply these equations to the magnetic field inside a magnetic multipole device which has the $z$-axis as its central axis. Our region of interest $G$ is, in cilindrical coordinates, given by: $G$ : $0 \leq r<R,-\pi \leq \varphi \leq \pi,-\infty<$ $z<\infty$, with boundary $\Gamma: r=R$. Here, $R>0$ is a convenient maximal radius of the multipole device, e.g. the aperture radius. We assume $u$ to be known at $\Gamma$, and introduce dimensionless coordinates $r *=r / R, z^{*}=z / R$. The potential problem for $u$ is then given by (we drop the stars for convenience):

\footnotetext{
${ }^{1}$ Eindhoven University of Technology, Den Dolech 2, P.O.Box 513, 5600 MB Eindhoven, The Netherlands, Department of Mathematics and Computer Science

${ }^{2}$ Eindhoven University of Technology, Department of Applied Physics

${ }^{3}$ FOM Institute for Plasma Physics "Rijnhuizen", P.O.Box 1207, 3430 BE Nieuwegein, The Netherlands
}

$$
\left\{\begin{array}{l}
\frac{\partial^{2} u}{\partial r^{2}}+\frac{1}{r} \frac{\partial u}{\partial r}+\frac{1}{r^{2}} \frac{\partial^{2} u}{\partial \varphi^{2}}+\frac{\partial^{2} u}{\partial z^{2}}=0 \quad \text { on } G \\
u(1, \varphi, z)=U(\varphi, z) \quad \text { on } \Gamma .
\end{array}\right.
$$

In order to have a unique solution to this problem, we have to impose the additional conditions

$$
\begin{cases}\lim _{z \rightarrow \pm \infty} u(r, \varphi, z)=C_{ \pm}<\infty, & 0 \leq r \leq 1, \\ \lim _{|z| \rightarrow \infty} u_{z}(r, \varphi, z)=0, & 0 \leq r \leq 1 .\end{cases}
$$

Here, $C_{+}$and $C_{-}$are constants such that $\lim _{z \rightarrow \pm \infty} u(r, \varphi, z)=C_{ \pm}$. For non-solenoidal fields (no $\varphi$-independent terms), we have $C_{ \pm}=0$. In practical cases, the conditions (5) will always be satisfied.

\section{FIELD DESCRIPTION}

The general solution to (4) can be expanded into $2 m$-pole contributions [1]:

$$
u=\sum_{m=0}^{\infty} J_{m}\left(r \frac{d}{d z}\right)\left(A_{m}(z) \cos (m \varphi)+B_{m}(z) \sin (m \varphi)\right),
$$

while the associated vector potential $\vec{A}$ is given by:

$$
\begin{aligned}
& A_{r}=\sum_{m=1}^{\infty} J_{m+1}\left(r \frac{d}{d z}\right)\left(B_{m} \cos (m \varphi)-A_{m} \sin (m \varphi)\right), \\
& A_{\varphi}=\sum_{m=0}^{\infty} J_{m+1}\left(r \frac{d}{d z}\right)\left(A_{m} \cos (m \varphi)+B_{m} \sin (m \varphi)\right), \\
& A_{z}=\sum_{m=1}^{\infty} J_{m}\left(r \frac{d}{d z}\right)\left(-B_{m} \cos (m \varphi)+A_{m} \sin (m \varphi)\right) .
\end{aligned}
$$

The functions $A_{m}(z)$ and $B_{m}(z)$ are to be determined from the boundary conditions at $r=1$. Let $V_{m}(z)$ and $W_{m}(z)$ denote the Fourier coefficients of $U(\varphi, z)$, then

$$
J_{k}\left(r \frac{d}{d z}\right) A_{m}(z)=\int_{-\infty}^{\infty} g_{m}^{k}(r, z-\zeta) V_{m}(\zeta) d \zeta .
$$

A similar result holds for $J_{k}\left(r \frac{d}{d z}\right) B_{m}(z)$. The basic funcion $g_{m}^{k}(r, z)$ is given by

$$
g_{m}^{k}(r, z)=\frac{1}{2 \pi} \int_{-\infty}^{\infty} \frac{I_{k}(\omega r)}{I_{m}(\omega)} i^{k-m} e^{i \omega z} d \omega .
$$

Any field-related quantity can be expressed in the same manner as the potentials by differentiating or integrating this basic function. 


\section{FITTING THE MULTIPOLE FIELD}

\subsection{Boundary conditions known}

In practice, we obtain approximations of $V_{m}$ or its derivatives by interpolating a discrete set of measurements by a piecewise constant or linear function. First, assume $V_{m}$ is piecewise constant. Then there are pairs $\left(\lambda_{i}, z_{i}\right)$ with $\sum_{i} \lambda_{i}=0$ such that $U_{m}^{\prime}=\sum_{i} \lambda_{i} \delta\left(z-z_{i}\right)$. Then, after integration,

$$
J_{k}\left(r \frac{d}{d z}\right) A_{m}(z)=\sum_{i} \lambda_{i} G_{m}^{k}\left(r, z-z_{i}\right),
$$

where $G_{m}^{k}(r, z)=\int_{0}^{z} g_{m}^{k}(r, \zeta) d \zeta$. If $V_{m}$ is supposed to be piecewise linear, then $U_{m}^{\prime \prime}=\sum_{i} \lambda_{i} \delta\left(z-z_{i}\right)$, in which case we need to replace $G_{m}^{k}(r, z)$ by $\tilde{G}_{m}^{k}(r, z)=$ $\int_{0}^{z} G_{m}^{k}(r, \zeta) d \zeta$. As was shown above, related quantities can easily be derived by replacing the functions $G_{m}$ and $\tilde{G}_{m}$ by functions related to these quantities, while retaining the pairs $\left(\lambda_{i}, z_{i}\right)$.

The values for $\lambda_{i}$ are obtained from measurements. For example, assume $B_{z}$ has been measured at the points $\left(1, \varphi_{j}, w_{i}\right)$. The Fourier coefficients of $B_{z}(1, \varphi, z)$ are $U_{m}^{\prime}$ and $W_{m}^{\prime}$; these are obtained from the measurements using Fourier series theory. Employing a piecewise constant approximation for $U_{m}^{\prime}$, we write $\lambda_{i}=U_{m}^{\prime}\left(w_{i+1}\right)-U_{m}^{\prime}\left(w_{i}\right)$, and $a_{m}(r, z)=\sum_{i} \lambda_{i} \tilde{G}_{m}\left(r, z-z_{i}\right)$, where $z_{i}=\frac{1}{2}\left(w_{i+1}-\right.$ $\left.w_{i}\right)$. Measurements of other components can be treated in the same way.

It should be noted that, in order to determine the $2 \mathrm{~m}$ pole contribution, one needs measurements performed at $2 m$ different angles $\varphi_{i}$.

The errors in $a_{m}(r, z)$ are limited by those in $V_{m}$ :

$$
\sup _{z \in \mathbb{R}}\left|\delta a_{m}(r, z)\right| \leq r^{m} \sup _{z \in \mathbb{R}}\left|\delta V_{m}(z)\right|
$$

This justifies the use of the given approximations.

\subsection{Boundary conditions unknown}

Expressions for the potential can also be obtained in case measurements were performed at points not on the surface $r=1$. Assume measurements of $u(r, \varphi, z)$ were taken at the points with scaled coordinates $\left(r_{k}, \varphi_{i}, w_{j}\right)$, $k=1, \ldots, M, i=1, \ldots, P, j=1, \ldots, N$. In this case, we are able to fit at most $M$ different multipole contributions simultaneously; in most cases, the $2 m$-pole contributions corresponding to $m=1, \ldots, M$, will be fitted. If less than $M$ multipole coefficients are fitted, the remaining data can be used to improve the statistics of the fit.

As an example, assume that $u$ contains dominant normal-oriented quadrupole and sextupole contributions, and that all higher order contributions are negligible:

$$
u(r, \varphi, z)=a_{2}(r, z) \cos (2 \varphi)+a_{3}(r, z) \cos (3 \varphi) .
$$

The coefficients $a_{2}$ and $a_{3}$ are then approximated by

$$
\begin{aligned}
a_{2}(r, z ; \lambda) & \doteq \sum_{l=1}^{N} \lambda_{l} G_{2}\left(r, z-z_{l}\right) \\
a_{3}(r, z ; \mu) & \doteq \sum_{l=1}^{N} \mu_{l} G_{3}\left(r, z-z_{l}\right)
\end{aligned}
$$

where $z_{l}=\frac{1}{2}\left(w_{l}+w_{l+1}\right)$. This corresponds to a piecewise constant approximation of the multipole coefficients of $u$ at $r=1$. We denote the measured value of $u$ at $\left(r_{k}, \varphi_{i}, w_{j}\right)$ by $f_{k i j}$, and define the quantity $M(\lambda, \mu)$ by:

$$
M(\lambda, \mu)=\sum_{k=1}^{M} \sum_{i=1}^{P} \sum_{l=1}^{N}\left(f_{k i j}-u\left(r_{k}, \varphi_{i}, w_{j}\right)\right)^{2} .
$$

The optimal values for $\lambda, \mu$ are then obtained by minimizing $M(\lambda, \mu)$ under the conditions $\sum_{l} \lambda_{l}=\sum_{l} \mu_{l}=0$. Again, knowledge of the values for $\lambda, \mu$ completely determines the corresponding multipole contribution.

There are a few remarks to be made concerning this example:

- Measurements at at least two different angles are needed to fit both normal and skew multipole contributions.

- A surplus of angles and/or radii can be used to improve on statistics.

- The $w_{i}$-values at which signal is dominant over noise should be used to define $M(\lambda, \mu)$; the remaining $w_{i^{-}}$ values only serve to improve on statistics.

- Further improvement on the statistics can be obtained by using a weighted average for the sum of squares in the definition of $M(\lambda, \mu)$.

It should also be noted that the former method yields better results for individual multipole contributions, while the latter yields a better approximation of the total field, distributing higher order multipole contributions among the lower order multipole coefficients.

\section{TRANSFER FUNCTIONS}

\subsection{Numerical calculation}

Having obtained a complete field description in terms of field measurements, we show how to obtain the transfer function from numerical integration of the equations of motion. The system of Hamiltonian equations of motion for a charged particle in the field can be written in the following form:

$$
\left(\underline{q}^{\prime}, \underline{p^{\prime}}\right)(z)=\underline{f}\left(\underline{q}, \underline{p}, \vec{A}(z, \underline{q}), \frac{\partial \vec{A}}{\partial \vec{x}}(z, \underline{q})\right) .
$$

Here, $\underline{q}=(r, \varphi, t), \underline{p}=\left(p_{r}, p_{\varphi}, p_{z}\right)$, and $\vec{x}=(r, \varphi, z)$. 
As derived in the previous section, the components of $\vec{A}$ and their partial derivatives all take the form as in (7).

The system (8) will be solved by means of a finite difference method. The discrete version of (8) reads:

$$
\begin{aligned}
& (\underline{q}, \underline{p})\left(w_{i+1}\right)=(\underline{q}, \underline{p})\left(w_{i-1}\right)+\left(w_{i+1}-w_{i-1}\right) \times \\
& \quad \times \underline{f}\left(\underline{q}\left(w_{i}\right), \underline{p}\left(w_{i}\right), \vec{A}\left(w_{i}, \underline{q}\left(w_{i}\right)\right), \frac{\partial \vec{A}}{\partial \vec{x}}\left(w_{i}, \underline{q}\left(w_{i}\right)\right)\right) .
\end{aligned}
$$

We apply the initial condition $(\underline{q}, \underline{p})\left(w_{0}\right)=\left(\underline{q}_{0}, \underline{p}_{0}\right)$, and calculate $(\underline{q}, \underline{p})\left(w_{1}\right)$ from

$$
\begin{aligned}
(\underline{q}, \underline{p})\left(w_{1}\right)= & (\underline{q}, \underline{p})\left(w_{0}\right)+\left(w_{1}-w_{0}\right) \times \\
& \times \underline{f}\left(\underline{q}_{0}, \underline{p}_{0}, \vec{A}\left(w_{0}, \underline{q}_{0}\right), \frac{\partial \vec{A}}{\partial \vec{x}}\left(w_{0}, \underline{q}_{0}\right)\right) .
\end{aligned}
$$

We then find $\left(\underline{q}_{f}, \underline{p}_{f}\right)$ by repeated application of (9), described in terms of $\left(q_{0}, p_{0}\right)$ and the field measurements. By varying $\left(q_{0}, p_{0}\right)$, we can calculate the complete transfer function of the device, directly in terms of these measurements.

Since the steps of this finite difference method are located precisely at the points $z=w_{i}$, where the field measurements were performed, we find that there are no interpolation errors in the values for $\vec{A}$ used in the calculations. By optimizing the interpolation of the boundary values at $r=1$, such that not only the boundary values, but also their $z$-derivatives are matched at the points $z=w_{i}$, we can also remove interpolation errors from the values of the partial derivatives of $\vec{A}$ for better results.

Note that for the numerical scheme, we need a best possible overall approximation of the magnetic vector potential, so the least squares method is preferred over direct integration of the boundary values in this case.

\subsection{Analytical results}

In the past, aberration coefficients for the transfer functions of many magneto-optical devices have been derived analytically. (See Smith [2], Lee-Whiting [3], Matsuda \& Wollnik [4], Nakabushi \& Matsuo [5].) Often, these coefficients are expressed in the on-axis $r$-derivatives of the field of such devices. The methods presented here can be used to obtain these gradients directly from field measurements away from the $z$-axis.

By expanding the functions $g_{m}$ into powers of $r$, one can expand the coefficients $a_{m}$ and $b_{m}$ into powers of $r$. For example:

$$
\begin{aligned}
a_{m}(r, z) & =\sum_{l=0}^{\infty} \alpha_{m l}(z) r^{m+2 l} \\
\alpha_{m l}(z) & =\int_{-\infty}^{z} g_{m l}(z-\zeta) V_{m}(\zeta), \\
g_{m l}(z) & =\frac{1}{4^{l} l !(m+l) ! \pi} \int_{0}^{\infty} \frac{\omega^{m+2 l}}{I_{m}(\omega)} \cos (\omega z) d \omega .
\end{aligned}
$$

The $z$-dependent coefficients of these power series and their derivatives are used in the expressions for the aberra- tion coefficients. The aberration coefficients for a normaloriented magnetic quadrupole, for example, are all expressed in terms of the quantity $k_{2}(z)$ and its derivatives, which are, for a particle with charge $q$ and momentum $p_{0}$, given by

$$
\left(k_{2}^{2}\right)^{(l)}(z)=\frac{q}{p_{0}} 4^{l} l !(l+2) ! \alpha_{2, l}(z) .
$$

From this, we find that we can avoid measuring the onaxis $r$-derivatives of the magnetic field, and obtain $k_{2}$, and thus the aberration coefficients, directly from measuring the magnetic field away from the $z$-axis, which is easier. The same holds for the aberration coefficients corresponding to higher order multipole contributions.

Note that, in order to calculate the various aberration coefficients properly, we need a best possible approximation of the individual multipole coefficients, so direct integration of the boundary values is the preferred method to obtain them.

\section{CONCLUSIONS}

The magnetic field inside a magnetic multipole, and its harmonic scalar and vector potentials, have been explored in the area $0 \leq r<1$ and $-\infty<z<\infty$. The various multipole contributions to these quantities have been fitted using field measurements at the boundary $r=1$ and shifted basic functions. An alternative least-squares method has been developed for measurements not at the boundary. The same set of measurements and shiftings can be used to fit many field-related quantities.

The developed procedure is independent of the exact form of the boundary conditions and can be used to fit the field of one device or various consecutive devices.

The procedure works for any order multipole contribution, but will be most useful for lower order multipole contributions, since higher order multipole contributions are more difficult to obtain from measurements and their effect on particle trajectories will often be small.

A numerical method for calculating the transfer functions from the field description has been derived. The field description has also been applied to existing analytical results. The least squares method is preferred in the first, direct integration of the boundary values in the second case.

\section{REFERENCES}

[1] R. Trines et al., EPAC-98 Conf. Proc. (1998), p. 1969.

[2] D.L. Smith, Nucl. Instr. and Meth. 79 (1970), p. 144; G.E. Lee-Whiting, Nucl. Instr. and Meth. 99 (1972), p. 609.

[3] G.E. Lee-Whiting, Nucl. Instr. and Meth. 83 (1970), p. 232.

[4] H. Matsuda \& H. Wollnik, Nucl. Instr. and Meth. 103 (1972), p. 117.

[5] H. Nakabushi \& T. Matsuo, Nucl. Instr. and Meth. 198 (1982), p. 207. 